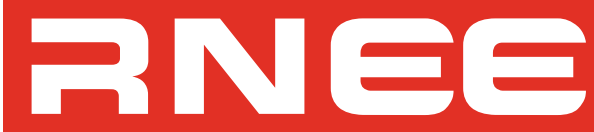

Revista Nicolaita de Estudios Económicos

Nueva Época

Instituto de Investigaciones Económicas y Empresariales
Universidad Michoacana de San Nicolás de Hidalgo

Volumen XVI, Número 1, Enero - Junio 2021, pp. 133 - 147

ISSN (Print): 1870 - 5464 ISSN (On-line): 2007 - 9877

DOI: 10.33110/rnee.v16i1.325

(Recibido: 26/agosto/2020; Aceptado: 11/mayo/2021)

\title{
La productividad de los factores en el cambio estructural de la economía de México en los años 1950: el régimen de crecimiento
}

Factor productivity and structural change in the economy of Mexico in the 1950s: The growth regime

Fidel Aroche Reyes a *

\section{Resumen}

Este trabajo tienen el propósito de estudiar los cambios en la estructura productiva mexicana con relación con el empleo de los factores productivos y su productividad durante los años 1950. Estos elementos se emplean para calcular los resultados de unos indicadores que caracterizan el "régimen de crecimiento" de la economía en un período particular y que se emplean aquí para este propósito. El modelo Insumo-Producto es el marco de referencia para tal metodología de análisis. El principal componente de la base de datos son las Matrices de Insumo-Producto de 1950 y 1960 desagregadas a 30 ramas homogéneas.

Palabras claves: desarrollo económico, industrialización, estructura productiva, insumo-producto, México

Clasificación JEL: O14, O47, O54, C67

\begin{abstract}
This paper studies the changes of the Mexican productive structure in relation to the productive factors' employment and their productivity between 1950 and 1960, by means of the results of two indicators helpful to characterise the "growth regime" that the economy follows a particular period. The Input-Output model frames the construction of such indicators as well as the interpretation of the results. The main component of the database are the 1950 and 1960 Input-Output tables disaggregated into 30 homogeneous industries.
\end{abstract}

Keywords: economic development, industrialization, productive structure, input-output, Mexico

JEL Codes: O14, O47, O54, C67

\footnotetext{
${ }^{a}$ Facultad de Economía, Universidad Nacional Autónoma de México

*Autor de correspondencia
} 


\section{Introducción}

Este trabajo explora algunos aspectos del cambio estructural en relación con el empleo de los factores y con la definición del concepto de "régimen de crecimiento" que experimentó la economía mexicana en el decenio que va de 1950 a 1960. Desde el punto de vista de la historia económica del país, este período es de particular interés por la rápida expansión industrial y el crecimiento de la economía en general, que quizás no ha sido analizado suficientemente hasta ahora, por lo menos desde el punto de vista de la estructura productiva y su transformación.

Durante el decenio que va de 1950 a 1960 la economía se transformó aceleradamente, mientras ocurrían dos fenómenos de interés para esta investigación; primero, se creaban y expandían diversas actividades manufactureras y de servicios y, segundo, se trasladaba la fuerza de trabajo desde la agricultura y otras actividades de baja productividad hacia la industria y los servicios de mayor productividad (Unikel y Torres, 1970). Estas actividades crecían más aceleradamente que las ramas rurales o la minería, cambiando no solo la composición por ramas del producto, sino también la manera en que se relacionaban éstas entre sí. Ocurrió entonces que el valor agregado total de la economía mexicana creció al 7\% promedio anual, mientras que el valor de los insumos intermedios aumentó en 8\%, las importaciones intermedias en 5\% por año; asimismo, el valor de los insumos intermedios producidos en México, representan el 28\% del valor bruto de la producción en 1950 y el $32 \%$ en 1960, sustituyendo los de origen nacional a los importados (Aroche, 2020). El sistema económico se hizo más complejo y se diversificó la oferta.

El desarrollo económico en general puede atribuirse a dos causas principales, a la acumulación de factores productivos en las distintas ramas y al empleo más eficiente de éstos, merced de la adopción de tecnologías nuevas. Si bien ambos fenómenos ocurren desigualmente entre las distintas ramas, ambos operan simultáneamente y generan cambios en el peso de cada una de las actividades productivas, así como en la manera en que los productores intercambian bienes (que cada uno emplea en la línea de producción). Por otra parte, estas causas mencionadas operan en importancia diversa en cada período de la historia económica, que puede entonces caracterizarse según si el crecimiento se atribuye más al crecimiento de la productividad o a la acumulación de factores (Antonelli y de Liso, 2000; Krugman, 1994).

Este trabajo explora el régimen de crecimiento que caracterizó a la economía mexicana en la década de 1950 como un todo y en cada una de las ramas en las que se desagrega ésta. Para ello emplea principalmente dos indicadores propuestos por Aroche (2020), que cuantifican la intensidad relativa de la acumulación de los factores y el crecimiento de su productividad. El modelo Insumo-Producto (IP) proporciona el marco de referencia para el estudio de la economía, entendida como un sistema de ramas interrelacionadas, así como de sus elementos individuales o de subconjuntos de éstos, es decir, de los sectores económicos, siempre en con la perspectiva de que forman parte de este sistema.

El componente principal de la base de datos empleado son las Matrices de Insumo-Producto de 1950 y 1960, hechas compatibles y editadas juntas bajo el título "Bases Informativas para la Utilización del Modelo de Insumo-Producto" (2 tomos) en 1980 por la Dirección General de Estadística, perteneciente a la Coordinación General de los Servicios Nacionales de Estadística, Geografía e Informática de la Secretaría de Programación y Presupuesto. Esta publicación recoge las matrices de Insumo-Producto (IP) de 1950, 1960 y 1970, publicadas respectivamente por el Banco de México, la Nacional Financiera y la Secretaría de Economía, en 1958; por el Banco de México en 1966 y por la Secretaría de Programación y Presupuesto, el Banco de México y la Organización de las Naciones Unidas en 1979 (Dirección General de Estadística, 1980), además de presentar 
unas versiones homogeneizadas de las tres tablas a 30 sectores a precios corrientes de cada año y a precios constantes de 1970. Hasta donde mi conocimiento alcanza, a la fecha tal base de datos se ha empleado poco (si algo) en el estudio de la economía mexicana y su evolución en esa época de gran dinamismo económico, a pesar de las evidentes posibilidades que abre su existencia a la investigación. Este trabajo se propone contribuir subsanar en algo tal estado de cosas, llegando a conclusiones principalmente a partir de los resultados de dos indicadores aplicados en un ejercicio que involucra a las tablas de 1950 y 1960.

\section{Empleo, productividad, régimen de crecimiento}

Como se establece arriba, durante la década que va entre 1950 y 1960 la economía creció aceleradamente en medio de un gran impulso al crecimiento de la industria y de los servicios modernos, cambiando también la estructura de relaciones de intercambio entre las ramas, así como la composición del producto y de los factores; en el sentido del marco teórico de referencia empleado aquí, se produjo un sensible cambio tecnológico y de la estructura productiva. La economía se desarrolló rápidamente en ese período (Aroche, 2020).

De acuerdo con datos del INEGI (1994), el empleo se expandió de 8.272.093 trabajadores en 1950 a 11.332.016 diez años más tarde, es decir, un 37\% ó 3,2\% en promedio anual; como punto de comparación, la población total creció un 35\%, de 25,791 millones a 34,923 millones, es decir, al promedio anual de 3,1\%. Aquella variable creció marginalmente por encima de la población, es decir, a un ritmo insuficiente para que el sector moderno de la economía absorbiera a la totalidad de la población excedente, empleada ineficazmente en actividades de baja productividad (Lewis, 1954). Así, la población ocupada total representa una proporción apenas superior a un tercio del total en ambos períodos, lo que se explica también por el acelerado crecimiento poblacional (Presidencia de la República, 1963; Unikel y Torres, 1970).

Por supuesto, la composición del empleo se modificó entre los sectores y entre las ramas, pero quizás no tanto como podría esperarse en un modelo teórico de rápida industrialización de un país subdesarrollado. Por ejemplo, de acuerdo con Lewis (1954 y 1979) o Ranis y Fei (1961), como producto de esa industrialización, la fuerza de trabajo se desplaza desde el sector "tradicional" al "moderno", que en México en esa época pueden identificarse respectivamente con los sectores rurales, tales como el agropecuario, la pesca y la silvicultura y algunos servicios, por un lado y -por otro- los sectores modernos corresponden con la industria y algunos otros servicios, ligados a la actividad industrial. También en ese período la minería era una actividad con altos requerimientos de capital, escaso empleo directo y producción de alto valor que perdía importancia en la composición del producto, puesto que mostraba la tasa de crecimiento menor entre todos los sectores económicos (Aroche, 2020). De acuerdo con los modelos de desarrollo, tal desplazamiento de la ocupación hacia las ramas modernas favorece la urbanización y es un factor para el crecimiento de la productividad factorial y de los ingresos en la economía como un todo, mientras es un soporte para la constitución del mercado interno para las manufacturas cuya producción se expande. Aún más, si esta migración supera al crecimiento poblacional, la expansión de la productividad será aún mayor (CEPAL, 1951).

En México el empleo en las actividades extractivas (agropecuarias y minería) alcanza en 1950 un $59,5 \%$ y en 1960 un 55,7\% del total; los servicios representan 25,7 y $26,8 \%$ para cada uno de esos años. La industria total llega al 14,8 y la manufacturera absorbe el 11,8\% en 1950; en 1960 las cifras son respectivamente el 17,7 y 13,7\%. Es desde luego problemático calificar el crecimiento del empleo en los servicios en términos de la modernización de la economía, puesto que allí se encuentra una combinación de actividades funcionales a la creciente industria, junto con actividades de refugio de mano de obra que no accede al mercado de trabajo 
formal. La ocupación en las ramas de Comercio y Otros servicios, además de la población ocupada en actividades insuficientemente especificadas pasó del 21,4 al 20,4\% del total y es allí donde probablemente se contabilicen la mayor parte del empleo en las actividades informales.

La población ocupada creció al 2,45\% promedio anual en la década de 1950 en el sector primario, al $3,83 \%$ en la minería, al 5,08\% en la industria y al 3,63\% en los servicios (ver Cuadro 1). Es decir, puede esperarse que el empleo se haya "urbanizado" en el sentido de que la industria muestra los mayores ritmos de crecimiento y de hecho la población rural disminuyó como porcentaje del total del 57,4\% al 49,3\%. No obstante, ocurren al menos tres fenómenos que merece la pena mencionar, por una parte, México es en 1950 un país eminentemente rural, carácter que no podía cambiar en los siguientes diez años al ritmo de crecimiento del valor agregado ocurrido -como ya se ha discutido. Enseguida, la población creció aceleradamente también, de modo que parte de la población que emigró del campo a la ciudad industrializante fue sustituida por nuevos trabajadores que se incorporaban al mercado de trabajo en el campo mismo, de modo que la modernización de los sectores rurales ha sido más lenta de lo esperado. Por otra parte, de acuerdo con datos del Centro Latinoamericano y Caribeño de Demografía (CELADE), la población económicamente activa como porcentaje del total disminuyó en la década de estudio del $46.8 \%$ al $41.5 \%$, lo cual se asocia al crecimiento más que proporcional de los rangos de población más joven, sin acceso al mercado de trabajo (no se registra el fenómeno del envejecimiento de la población).

Cuadro 1 Empleo sectorial: Número de empleados (participación en el total)

\begin{tabular}{lrrr}
\hline & 1950 & 1960 & $\begin{array}{c}\text { Tasa de crecimiento } \\
\text { anual medio }\end{array}$ \\
\hline Sector agropecuario & $4823901(58.32 \%)$ & $6143540(54.21 \%)$ & $2.45 \%$ \\
Minería & $97143(1.17 \%)$ & $141530(1.25 \%)$ & $3.83 \%$ \\
Industria & $1222020(14.77 \%)$ & $2005813(17.70 \%)$ & $5.08 \%$ \\
Servicios & $2129029(25.74 \%)$ & $3041133(26.84 \%)$ & $3.63 \%$ \\
Total & $8272093(100 \%)$ & $11332016(100 \%)$ & $3.20 \%$ \\
\hline
\end{tabular}




\section{Productividad laboral}

Como discute Aroche (2021), el valor agregado total en 1950 llegó a \$106.661.300.000,00, mientras que en 1960 la cifra alcanzó \$201.258.300.000,00 (ambos en pesos constantes de 1970), es decir, entre esos dos años ascendió en casi $89 \%$, a una tasa de crecimiento anual media de 6,6\%, de donde el VA por trabajador pasó de $\$ 12.894,11$ a $\$ 17.760,15$, es decir, se elevó en $\$ 4.866,04$ ó en $37.7 \%$. La tasa de crecimiento anual media para esta variable fue de $3,3 \%$, marginalmente por encima del crecimiento del empleo.

El Cuadro 1 muestra el empleo por rama y su crecimiento promedio anual entre 1950 y 1960. La rama de madera y corcho, junto con las actividades insuficientemente especificadas muestran reducciones absolutas, pero hay otras ramas que pierden peso debido a que experimentan reducidos ritmos de expansión, como la minería, los productos del cuero o los textiles. Básicamente se trata de ramas ya maduras para 1950 y que -como se ha dicho ya- crecen por debajo del promedio, probablemente ante mercados nacionales saturados, además de que no se transforman en exportadoras para continuar su crecimiento. Por último, se encuentran diez ramas cuyo empleo crece a tasas muy altas relativas al sistema económico (superiores al 7\% anual promedio) pero que inicialmente muestran participaciones reducidas en el total, de modo que no consiguen superar a las actividades tradicionales (como la agricultura) y determinar la dinámica de la economía. Se trata de sectores productores de bienes que emplean tecnologías más elaboradas, así como de servicios que también avanzan con la formalización del empleo como los restaurantes y hoteles, rama que muestra la mayor tasa de crecimiento del empleo de la economía.

El Cuadro 2 muestra el VA por trabajador por rama y recoge también el crecimiento del VA total por rama; el crecimiento de aquel es sistemáticamente menor que el de éste y el coeficiente de correlación entre las tasas de crecimiento es de 0.484 , de donde puede decirse que el producto se expande preferentemente por la agregación en el empleo de factores productivos en la economía, más que por incrementos de la eficiencia de la producción y del empleo factorial. Por otra parte, en 1950 aparecen 15 ramas con relaciones que más que duplican al promedio de toda la economía (\$12.894,11); para 1960 solo cinco ramas cumplen con esa condición, es decir, que tiende a igualarse la productividad factorial entre los sectores. Sin embargo, ocho ramas superan el crecimiento de la productividad del empleo en la economía, mientras que, en cuatro manufactureras (productos alimenticios, calzado y prendas de vestir, madera y corcho, metálicas básicas), la construcción y en dos ramas de servicios, (transporte y restaurantes y hoteles) la productividad del trabajo disminuye; el empleo en la rama de la madera y corcho decrece, además del valor agregado. En la rama de productos alimenticios y metálicas básicas el empleo crece por encima del total, al igual que el VA, si bien aquel supera a éste; por último, en la rama de calzado y prendas de vestir, al igual que en la construcción, transportes y restaurantes y hoteles. El VA crece lentamente, mientras el empleo supera a este ritmo. 


\begin{tabular}{|c|c|c|c|c|c|c|}
\hline & & \multicolumn{2}{|c|}{1950} & \multicolumn{2}{|r|}{1960} & \multirow{2}{*}{$\begin{array}{c}\text { T. de C anual } \\
\text { promedio }\end{array}$} \\
\hline & & Empleo & Participación & Empleo & Participación & \\
\hline 1 & Agricultura & 2868614 & $34.68 \%$ & 3676249 & $32.44 \%$ & $2.51 \%$ \\
\hline 2 & Ganadería & 1688781 & $20.42 \%$ & 2101395 & $18.54 \%$ & $2.21 \%$ \\
\hline 3 & Silvicultura, caza y pesca & 266506 & $3.22 \%$ & 365896 & $3.23 \%$ & $3.22 \%$ \\
\hline 4 & Minería & 57050 & $0.69 \%$ & 61068 & $0.54 \%$ & $0.68 \%$ \\
\hline 5 & Petróleo y coque & 40093 & $0.48 \%$ & 80462 & $0.71 \%$ & $7.21 \%$ \\
\hline 6 & Productos alimenticios & 276789 & $3.35 \%$ & 487762 & $4.30 \%$ & $5.83 \%$ \\
\hline 7 & Bebidas & 52422 & $0.63 \%$ & 87851 & $0.78 \%$ & $5.30 \%$ \\
\hline 8 & Tabaco y sus productos & 26617 & $0.32 \%$ & 31752 & $0.28 \%$ & $1.78 \%$ \\
\hline 9 & Textiles & 131898 & $1.59 \%$ & 132613 & $1.17 \%$ & $0.05 \%$ \\
\hline 10 & Calzado y prendas de vestir & 114504 & $1.38 \%$ & 140027 & $1.24 \%$ & $2.03 \%$ \\
\hline 11 & Madera y corcho & 53632 & $0.65 \%$ & 38302 & $0.34 \%$ & $-3.31 \%$ \\
\hline 12 & Papel, cartón y sus productos & 16878 & $0.20 \%$ & 40515 & $0.36 \%$ & $9.15 \%$ \\
\hline 13 & Imprenta y editorial & 24695 & $0.30 \%$ & 37181 & $0.33 \%$ & $4.18 \%$ \\
\hline 14 & Cuero y sus productos & 24841 & $0.30 \%$ & 27009 & $0.24 \%$ & $0.84 \%$ \\
\hline 15 & Hule y sus productos & 11271 & $0.14 \%$ & 21852 & $0.19 \%$ & $6.84 \%$ \\
\hline 16 & Química farmacéutica & 61137 & $0.74 \%$ & 135625 & $1.20 \%$ & $8.29 \%$ \\
\hline 17 & Productos de minerales no metálicos & 33558 & $0.41 \%$ & 55725 & $0.49 \%$ & $5.20 \%$ \\
\hline 18 & Metálicas básicas & 37355 & $0.45 \%$ & 91180 & $0.80 \%$ & $9.33 \%$ \\
\hline 19 & Productos metálicos & 26954 & $0.33 \%$ & 53519 & $0.47 \%$ & $7.10 \%$ \\
\hline 20 & Maquinaria y equipo & 30487 & $0.37 \%$ & 63372 & $0.56 \%$ & $7.59 \%$ \\
\hline 21 & Equipo de transporte y automotriz & 34033 & $0.41 \%$ & 80470 & $0.71 \%$ & $8.99 \%$ \\
\hline 22 & Manufacturas diversas & 15471 & $0.19 \%$ & 31334 & $0.28 \%$ & $7.31 \%$ \\
\hline 23 & Construcción & 224512 & $2.71 \%$ & 408279 & $3.60 \%$ & $6.16 \%$ \\
\hline 24 & Electricidad & 24966 & $0.30 \%$ & 41443 & $0.37 \%$ & $5.20 \%$ \\
\hline 25 & Comercio & 684092 & $8.27 \%$ & 1075174 & $9.49 \%$ & $4.63 \%$ \\
\hline 26 & Transporte & 210592 & $2.55 \%$ & 356939 & $3.15 \%$ & $5.42 \%$ \\
\hline 27 & Comunicaciones & 18040 & $0.22 \%$ & 36958 & $0.33 \%$ & $7.44 \%$ \\
\hline 28 & Restaurantes y hoteles & 66024 & $0.80 \%$ & 179419 & $1.58 \%$ & $10.51 \%$ \\
\hline 29 & Servicios financieros & 67191 & $0.81 \%$ & 158548 & $1.40 \%$ & $8.96 \%$ \\
\hline 30 & Otros servicios & 728124 & $8.80 \%$ & 1152304 & $10.17 \%$ & $4.70 \%$ \\
\hline & No suficientemente especificado & 354966 & $4.29 \%$ & 81791 & $0.72 \%$ & $13.65 \%$ \\
\hline & Total & 8272093 & $100.00 \%$ & 11332016 & $100.00 \%$ & $3.20 \%$ \\
\hline
\end{tabular}

Fuente: Elaborado con los datos de las matrices de Insumo-Producto de 1950 y 1960 (Vide textum) 


\begin{tabular}{|c|c|c|c|c|c|}
\hline & & 1950 & 1960 & $\begin{array}{c}\text { T. de C } \\
\text { media anual }\end{array}$ & $\begin{array}{l}\text { Crec. Medio del } \\
\text { V A total por rama }\end{array}$ \\
\hline 1 & Agricultura & $4,456.19$ & $5,511.08$ & $2.15 \%$ & $4.71 \%$ \\
\hline 2 & Ganadería & $3,998.21$ & $4,567.20$ & $1.34 \%$ & $3.58 \%$ \\
\hline 3 & Silvicultura, caza y pesca & $4,637.80$ & $5,558.41$ & $1.83 \%$ & $5.11 \%$ \\
\hline 4 & Minería & $58,075.79$ & $74,112.48$ & $2.47 \%$ & $3.17 \%$ \\
\hline 5 & Petróleo y coque & $41,223.73$ & $61,653.94$ & $4.11 \%$ & $11.62 \%$ \\
\hline 6 & Productos alimenticios & $14,108.57$ & $15,462.05$ & $3.51 \%$ & $9.54 \%$ \\
\hline 7 & Bebidas & $27,206.15$ & $19,305.74$ & $-0.89 \%$ & $4.36 \%$ \\
\hline 8 & Tabaco y sus productos & $34,917.60$ & $31,196.71$ & $1.42 \%$ & $3.22 \%$ \\
\hline 9 & Textiles & $19,819.09$ & $21,334.86$ & $3.32 \%$ & $3.38 \%$ \\
\hline 10 & Calzado y prendas de vestir & $32,589.28$ & $25,197.70$ & $-0.04 \%$ & $1.99 \%$ \\
\hline & Madera y corcho & $28,529.85$ & $18,640.68$ & $-0.91 \%$ & $-4.19 \%$ \\
\hline 12 & Papel, cartón y sus productos & $12,697.10$ & $13,430.93$ & $3.14 \%$ & $12.58 \%$ \\
\hline 13 & Imprenta y editorial & $25,883.40$ & $24,909.17$ & $2.17 \%$ & $6.44 \%$ \\
\hline 14 & Cuero y sus productos & $13,123.41$ & $18,364.91$ & $6.07 \%$ & $6.96 \%$ \\
\hline 15 & Hule y sus productos & $15,499.48$ & $17,359.76$ & $3.73 \%$ & $10.83 \%$ \\
\hline 16 & Química farmacéutica & $12,666.68$ & $13,338.04$ & $3.10 \%$ & $11.65 \%$ \\
\hline 17 & Productos de minerales no metálicos & $28,026.13$ & $24,452.66$ & $1.18 \%$ & $6.44 \%$ \\
\hline 18 & Metálicas básicas & $21,769.31$ & $14,846.47$ & $-1.29 \%$ & $7.93 \%$ \\
\hline & Productos metálicos & $25,684.29$ & $20,417.45$ & $0.24 \%$ & $7.35 \%$ \\
\hline 20 & Maquinaria y equipo & $23,875.61$ & $22,642.31$ & $2.02 \%$ & $9.77 \%$ \\
\hline & Equipo de transporte y automotriz & $22,058.24$ & $20,612.43$ & $1.87 \%$ & $11.03 \%$ \\
\hline 22 & Manufacturas diversas & $23,283.00$ & $23,903.93$ & $2.84 \%$ & $10.36 \%$ \\
\hline 23 & Construcción & $32,212.53$ & $24,151.13$ & $-2.84 \%$ & $3.15 \%$ \\
\hline 24 & Electricidad & $24,405.19$ & $40,984.00$ & $5.32 \%$ & $10.80 \%$ \\
\hline 25 & Comercio & $34,108.57$ & $57,028.72$ & $5.27 \%$ & $10.14 \%$ \\
\hline 26 & Transporte & $85,096.00$ & $84,849.40$ & $-1.31 \%$ & $4.03 \%$ \\
\hline 27 & Comunicaciones & $29,683.67$ & $52,522.38$ & $0.19 \%$ & $7.64 \%$ \\
\hline 28 & Restaurantes y hoteles & $28,935.10$ & $49,177.38$ & $-0.22 \%$ & $10.28 \%$ \\
\hline 29 & Servicios financieros & $27,119.50$ & $51,932.24$ & $0.98 \%$ & $10.03 \%$ \\
\hline \multirow[t]{2}{*}{30} & Otros servicios & $20,126.50$ & $45,407.29$ & $3.14 \%$ & $4.50 \%$ \\
\hline & Total & $12,894.11$ & $17,760.15$ & $3,25 \%$ & $6.50 \%$ \\
\hline
\end{tabular}

Fuente: Elaborado con los datos de las matrices de Insumo-Producto de 1950 y 1960 (Vide textum) 
Los sectores agropecuarios muestran las menores productividades, de modo que en 1960 la minería es 16 veces más productiva que la ganadería, cada una siendo la rama con mayor y la menor relación producto por trabajador en esa década. No obstante, ambas presentan tasas de crecimiento menores al total, perdiendo peso en la composición del producto y el empleo. Es interesante que la productividad de las ramas manufactureras con menor tecnificación, como el cuero y sus productos, las bebidas, la madera y el corcho, el calzado y el vestido, entre otras, muestren altas relaciones de producto por unidad de trabajo, pero también menores tasas de crecimiento. Ocurre entonces un reacomodo de las ramas a favor de aquellas con menor productividad inicial, pero con mayores tasas de crecimiento en la variable. No obstante, como se aprecia a simple vista, no se cumple que las ramas con mayor crecimiento de la productividad sean aquellas con mayor expansión del VA. El crecimiento de muchas ramas se consigue preferentemente por la adición de factores, principalmente fuerza de trabajo que supera a otros factores productivos y a la producción misma.

El Cuadro 3 recoge la composición de la inversión (la suma de la formación bruta de capital y variación de existencias) y su tasa media de crecimiento por rama. Primero salta a la vista la inestabilidad de esta variable, algunas ramas muestran participaciones negativas en el total en el año 1950; enseguida, la rama de la construcción concentra la parte del león de esta variable. La fuente no lo menciona, pero es probable que el total de la actividad constructiva de la economía se haya atribuido a la rama proveedora; el comercio es otra rama con una participación muy por encima del resto. La participación del resto de las ramas en el total de la inversión tiende a uniformarse. El crecimiento de la inversión para la mayor parte de las ramas en esta década de 1950 es también muy alto, si bien para el total la tasa es razonable; es decir, la variable observa un comportamiento muy desigual entre los sectores, pero puede esperarse que haya mostrado un crecimiento vigoroso en el período a lo largo de toda la economía, confirmando la hipótesis mostrada antes: el crecimiento se explica por la acumulación de factores, si bien la correlación entre la tasa de crecimiento de la inversión y del valor agregado es negativa (0.51); asimismo, la relación entre el VA y la inversión se redujo durante la década a una tasa media de $-4.2 \%$, donde algunas actividades muestran tasas negativas de dos dígitos. Por supuesto, sería menester considerar el comportamiento del acervo de capital, antes de llegar a conclusiones sobre su relación con el VA. 

Existencias) Composición \% y Tasa media de crecimiento por rama \%

\begin{tabular}{|c|c|c|c|c|c|}
\hline & Rama & 1950 & 1960 & T. de C & Crec. Medio VA \\
\hline 1 & Agricultura & $3.69 \%$ & $4.28 \%$ & $10.68 \%$ & $4.71 \%$ \\
\hline 2 & Ganadería & $2.60 \%$ & $3.31 \%$ & $11.71 \%$ & $3.58 \%$ \\
\hline 3 & Silvicultura, caza y pesca & $0.02 \%$ & $0.04 \%$ & $14.28 \%$ & $5.11 \%$ \\
\hline 4 & Minería & $-0.69 \%$ & $0.42 \%$ & $\mathrm{ND}$ & $3.17 \%$ \\
\hline 5 & Petróleo y coque & $-0.25 \%$ & $0.68 \%$ & ND & $11.62 \%$ \\
\hline 6 & Productos alimenticios & $0.80 \%$ & $1.35 \%$ & $14.89 \%$ & $9.54 \%$ \\
\hline 7 & Bebidas & $0.20 \%$ & $0.22 \%$ & $10.07 \%$ & $4.36 \%$ \\
\hline 8 & Tabaco y sus productos & $0.07 \%$ & $0.09 \%$ & $13.02 \%$ & $3.22 \%$ \\
\hline 9 & Textiles & $1.43 \%$ & $0.78 \%$ & $2.66 \%$ & $3.38 \%$ \\
\hline 10 & Calzado y prendas de vestir & $0.64 \%$ & $1.45 \%$ & $18.37 \%$ & $1.99 \%$ \\
\hline 11 & Madera y corcho & $1.17 \%$ & $0.58 \%$ & $1.72 \%$ & $-4.19 \%$ \\
\hline 12 & Papel, cartón y sus productos & $0.01 \%$ & $0.16 \%$ & $40.82 \%$ & $12.58 \%$ \\
\hline 13 & Imprenta y editorial & $0.07 \%$ & $0.19 \%$ & $19.90 \%$ & $6.44 \%$ \\
\hline 14 & Cuero y sus productos & $0.49 \%$ & $0.19 \%$ & $-0.59 \%$ & $6.96 \%$ \\
\hline 15 & Hule y sus productos & $0.09 \%$ & $0.23 \%$ & $20.20 \%$ & $10.83 \%$ \\
\hline 16 & Química farmacéutica & $0.57 \%$ & $1.20 \%$ & $17.51 \%$ & $11.65 \%$ \\
\hline 17 & Productos de minerales no metálicos & $0.26 \%$ & $0.50 \%$ & $16.60 \%$ & $6.44 \%$ \\
\hline 18 & Metálicas básicas & $0.63 \%$ & $1.68 \%$ & $20.33 \%$ & $7.93 \%$ \\
\hline 19 & Productos metálicos & $0.78 \%$ & $2.37 \%$ & $21.89 \%$ & $7.35 \%$ \\
\hline 20 & Maquinaria y equipo & $2.40 \%$ & $2.44 \%$ & $9.25 \%$ & $9.77 \%$ \\
\hline 21 & Equipo de transporte y automotriz & $6.23 \%$ & $6.87 \%$ & $10.12 \%$ & $11.03 \%$ \\
\hline 22 & Manufacturas diversas & $0.24 \%$ & $0.46 \%$ & $16.37 \%$ & $10.36 \%$ \\
\hline 23 & Construcción & $71.71 \%$ & $53.25 \%$ & $5.86 \%$ & $3.15 \%$ \\
\hline 24 & Electricidad & $0.00 \%$ & $0.38 \%$ & ND & $10.80 \%$ \\
\hline 25 & Comercio & $6.19 \%$ & $14.44 \%$ & $18.70 \%$ & $10.14 \%$ \\
\hline 26 & Transporte & $0.07 \%$ & $0.29 \%$ & $26.46 \%$ & $4.03 \%$ \\
\hline 27 & Comunicaciones & $0.00 \%$ & $0.00 \%$ & ND & $7.64 \%$ \\
\hline 28 & Restaurantes y hoteles & $0.00 \%$ & $0.00 \%$ & ND & $10.28 \%$ \\
\hline 29 & Servicios financieros & $0.02 \%$ & $0.00 \%$ & $-100.00 \%$ & $10.03 \%$ \\
\hline \multirow[t]{2}{*}{30} & Otros servicios & $0.58 \%$ & $2.15 \%$ & $24.37 \%$ & $4.50 \%$ \\
\hline & Total & $100.00 \%$ & $100.00 \%$ & $9.06 \%$ & $6.50 \%$ \\
\hline
\end{tabular}

Fuente: Elaborado con los datos de las matrices de Insumo-Producto de 1950 y 1960 (Vide textum) 


\section{El régimen de crecimiento en los años 1950}

El desarrollo de las economías implica el cambio de la estructura del aparato productivo, es decir, cuando aparecen nuevas actividades, incrementándose la presencia de las ramas con mayor productividad factorial y modificándose la manera en que los sectores intercambian bienes entre sí y con los agentes oferentes de factores y demandantes de bienes finales, incluyendo al sector externo. Esto último involucra cambios en la tecnología que los sectores individuales emplean para producir (Aroche, 2005; Aroche, 2020). En el modelo IP ello se refleja en el cambio de la composición de los vectores del consumo y la demanda intermedios, por un lado y de la demanda final y del valor agregado, por el otro, así como en el valor de los coeficientes (y de los intercambios en valor a precios constantes).

Asimismo, los ingresos de las ramas pueden tornar más intensivos en la demanda final o en la intermedia, mientras que los gastos de aquellas también pueden incrementar su componente, alternativamente intermedio o factorial. A partir de esta observación se construye la hipótesis, según la cual la economía sigue un determinado régimen de crecimiento (Aroche, 2005 y 2020). Es decir, el producto de la economía se expande mediante dos caminos alternos, según el tipo de insumos donde se concentran las ganancias de la productividad; el primero se basa en la productividad creciente de los factores, en cuyo caso disminuye su empleo por unidad de producto, mientras la demanda por bienes intermedios (capital circulante) se incrementa (también por unidad de producto). Alternativamente, el uso de los factores de coste se hace más eficiente por unidad de producto (disminuye el coeficiente de consumo intermedio de la economía como un todo) y se incrementan entonces los coeficientes de factores empleados por unidad de producto.

Leontief (1963) y Carter (1970) caracterizan a una economía más desarrollada por la complejidad del sector productivo, asociada con la mayor división del trabajo y con un entramado de intercambios donde todas las ramas simultáneamente compran y venden manera directa o indirecta una mayor variedad de bienes que usan como insumos en la producción. Al mismo tiempo, en una economía desarrollada, los factores alcanzan mayor productividad -o bien- se emplean de manera más eficiente, en relación con economías menos complejas porque las actividades productivas consiguen mayores niveles de especialización, de donde los procesos de aprendizaje factorial son más completos y es más fácil producir e introducir innovaciones, como propone Smith (1773: 2011). Sin embargo, las economías pueden alternar los distintos estilos de crecimiento en distintos períodos, ya sea incrementando la productividad de los factores o del capital circulante, o bien, incrementando el empleo de cada elemento productivo (en distintas proporciones), mientras que pueden también complejizar su aparato productivo, incrementando la cantidad de intercambios entre los productores e incrementando el valor de tales intercambios por unidad de producto, o bien, pueden decrementar la cantidad y valor de los intercambios o, por último, modificar la cantidad de intercambios pero no su valor o viceversa. La economía sigue un régimen de crecimiento u otro, según la evolución de la tecnología y de la disponibilidad y empleo de los factores; en definitiva, siguiendo a la teoría neoclásica, según la evolución del régimen de precios relativos. En un marco teórico más amplio, la acumulación de capital determina la naturaleza y dirección del desarrollo de las economías, incluyendo de manera muy notable la incorporación del progreso técnico (Kaldor, 1967; Böhm y Punzo, 1999; Antonelli y de Liso, 2000; Avendaño y Perrotini, 2017).

A partir de los resultados presentados en este conjunto de trabajos, es razonable pensar que, durante la década de 1950, la economía mexicana se desarrolló, en el sentido de Leontief (1963), complejizando la división del trabajo entre los sectores, donde cada uno diversificó sus proveedores de bienes y al conjunto de sus compradores (al menos en algunos planos, dado que las ramas manufactureras no se convirtieron en 
exportadoras). El resto de esta sección explora el comportamiento de la estructura productiva mexicana durante la década de 1950 con relación a la hipótesis del régimen de crecimiento.

En esta línea de razonamiento, Aroche (2005 y 2020) propone dos indicadores que -juntos- ayudan a caracterizar al régimen de crecimiento que sigue una economía durante algún período determinado y que se emplean a continuación. El primero, $\beta_{j}$ mide la respuesta promedio de los coeficientes técnicos al cambio del valor agregado por trabajador en la rama $j$, durante un período de tiempo dado (comparando las matrices IP de dos años). Este indicador supone que el cambio técnico en esa rama $j$ provoca que la productividad del trabajo cambie en alguna dirección, teniendo además efectos sobre el crecimiento o decrecimiento de los coeficientes técnicos. Este indicador se expresa como:

$$
\beta_{j}=\left[\Delta a_{j} / \Delta v_{j}\right] \cdot\left[v^{0} / a_{j}^{0}\right]
$$

Donde $\Delta a_{j}$ es la diferencia entre el promedio de los coeficientes $a_{i j}$ en la columna $j$ entre los periodos 0 and $1 ; \Delta v_{j}$ es la diferencia entre los valores agregados por trabajador en esa rama $j$ en los dos períodos de referencia. $v_{j}^{0}$ and $a_{j}^{0}$ representa las variables correspondientes en el periodo 0 . Entonces, $\beta_{j}$ mide la respuesta promedio de los coeficientes al cambio en el valor agregado por trabajador en la rama $j$. De manera análoga, $\eta_{j}$ se define como sigue:

$$
\eta_{j}=\left[\Delta a_{j} / \Delta i_{j}\right] \cdot\left[i_{j}^{0} / a_{j}^{0}\right]
$$

$\Delta i$ es la diferencia entre las razones de la formación de capital fijo bruto por empleado entre las dos fechas 0 y $1, i_{j}^{0}$ es esa razón en la fecha 0 e $\eta_{j}$ mide la capacidad de respuesta de los coeficientes técnicos a la formación de capital fijo en la rama $j$. El decremento o incremento en la razón de la formación de capital fijo por trabajador cambia las condiciones técnicas de producción y provocará cambios en los coeficientes técnicos.

$\beta_{j}$ e $\eta_{j}$ son parámetros que permite caracterizar la senda por la que evolucionan las relaciones estructurales de la rama $j$, de acuerdo con el estilo de progreso técnico adoptado por $j$. Allí donde $\beta_{j}>\eta_{j}$ el cambio tecnológico favorece a la productividad de los factores sobre la formación bruta de capital y del empleo del trabajo; por el contrario, si $\beta_{j}<\eta_{j}$, el crecimiento de la rama está determinada por la acumulación de factores, más que por el crecimiento de su productividad. No obstante, sería posible que un $\beta_{j}$ mayor se asocie con el decrecimiento de los coeficientes técnicos, por ejemplo, si las importaciones reemplazan a los insumos de origen nacional, rompiéndose las cadenas productivas y la integración entre los productores nacionales. De acuerdo con los resultados presentados hasta acá, sin embargo, parece que en México en los años 1950 ocurre exactamente lo contrario: se favorece el empleo de los insumos producidos al interior de la economía.

El Cuadro 4 presenta los resultados para estos parámetros por rama, su promedio y los resultados para el total de la economía. Para este último caso, así como para 17 sectores, los parámetros $\beta_{\mathrm{j}}$ son mayores que $\eta_{\mathrm{j}}$; es decir, a primera vista, el crecimiento de la economía obedeció a un régimen que favorece la mayor productividad factorial (en preferencia a la acumulación de factores productivos). No obstante, conviene mirar los resultados con mayor atención, por ejemplo, se encuentran 13 sectores (del total de 30) donde el parámetro $\eta_{j}$ es nulo porque la diferencia entre la FBK de 1960 a 1950 es cero, mientras que en siete de estos sectores $\eta_{j}$ y $\beta$ es negativo, porque los diferenciales entre los coeficientes de 1960 y 1950 lo son; es decir, las entradas $a_{i j}$ se reducen en promedio en esas ramas. Ello implica que allí el VA por unidad de producto se incrementa, pero a cambio, esos 
sectores se aíslan relativamente del resto del aparato productivo. Sin embargo, la reducción de los coeficientes por unidad de producto da lugar a un empleo más eficiente de los insumos.

En una palabra, los cambios relativos de los parámetros son contradictorios en la economía mexicana. Por ello, es difícil proponer una tipología de los sectores donde ocurren determinados fenómenos que dan lugar a estos regímenes de crecimiento, puesto que algunas ramas manufactureras modernas comparten características con ramas primarias o con algunos servicios de distinta naturaleza. 


\begin{tabular}{|c|c|c|c|}
\hline & & $\beta j=[\Delta a j / \Delta v j]]^{*}[V j / a j]$ & $\mathrm{nj}=[\Delta \mathrm{aj} / \Delta \mathrm{vi}]^{*}[\mathrm{ij} / \mathrm{aj}]$ \\
\hline 1 & Agricultura & 0.13 & 0.00 \\
\hline & Ganadería & -0.41 & 0.00 \\
\hline 3 & Silvicultura, caza y pesca & 3.66 & 0.00 \\
\hline 4 & Minería & 4.88 & 0.00 \\
\hline 5 & Petróleo y coque & 1.13 & 0.00 \\
\hline 6 & Productos alimenticios & -4.70 & 0.00 \\
\hline & Bebidas & -3.20 & 0.00 \\
\hline 8 & Tabaco y sus productos & -2.75 & 0.00 \\
\hline & Textiles & -3.36 & 0.00 \\
\hline 10 & Calzado y prendas de vestir & -2.89 & -0.01 \\
\hline 11 & Madera y corcho & 1.07 & 0.08 \\
\hline 12 & Papel, cartón y sus productos & 3.27 & 0.00 \\
\hline 13 & Imprenta y editorial & -3.86 & 0.00 \\
\hline 14 & Cuero y sus productos & -0.79 & 0.00 \\
\hline 15 & Hule y sus productos & 8.10 & 0.08 \\
\hline 16 & Química farmacéutica & -0.65 & 0.00 \\
\hline 17 & Productos de minerales no metálicos & -2.42 & 0.00 \\
\hline 18 & Metálicas básicas & -1.38 & -0.11 \\
\hline 19 & Productos metálicos & -1.80 & -0.26 \\
\hline 20 & Maquinaria y equipo & -1.80 & -0.77 \\
\hline 21 & Equipo de transporte y automotriz & -3.48 & -3.91 \\
\hline 22 & Manufacturas diversas & 7.95 & 0.28 \\
\hline 23 & Construcción & -2.94 & -4.26 \\
\hline 24 & Electricidad & -0.23 & 0.00 \\
\hline 25 & Comercio & -0.54 & -0.02 \\
\hline 26 & Transporte & -69.21 & -0.21 \\
\hline 27 & Comunicaciones & 0.18 & 0.00 \\
\hline 28 & Restaurantes y hoteles & 0.57 & 0.00 \\
\hline 29 & Servicios financieros & -0.12 & 0.00 \\
\hline & Otros servicios & 0.51 & 0.00 \\
\hline & Total & -75.12 & -9.11 \\
\hline & Promedio & -2.50 & -0.30 \\
\hline
\end{tabular}

Fuente: Elaborado a partir de las matrices de Insumo-Producto de 1950 y 1960 (Vide textum) 


\section{Conclusiones}

Efectivamente, durante los años 1950 la economía mexicana creció aceleradamente y transformó su estructura productiva, en el sentido de que las relaciones entre las ramas productivas se modificaron como resultado del crecimiento desigual entre ellas y del cambio tecnológico desequilibrado, explicado por dos fenómenos; por una parte, a escala internacional la década de referencia se caracteriza por la innovación de los procesos productivos y la aparición de nuevos productos, favorecidos por el crecimiento económico generalizado y por la puesta en práctica de diversos adelantos desarrollados en los años de la II Guerra Mundial.

En el plano de la economía nacional, sin embargo, la inversión (la formación bruta de capital más la variación de existencias) es desigual entre los sectores, al igual que la incorporación de innovaciones. Por supuesto, los sectores presentan distinto grado de madurez y, por lo tanto, tienen distinto espacio para crecer dado el tamaño del mercado interno, en una economía internacional que dificulta las exportaciones de manufacturas de los países menos desarrollados.

Este trabajo ha demostrado que el crecimiento de la economía mexicana en la década de 1950 está apoyado en la expansión de la productividad factorial, además de la adición de factores productivos. Es decir, como es evidente, la economía expandió el empleo tanto del factor trabajo como de la fuerza de trabajo; el cambio tecnológico, además, permitió que tal empleo haya sido más eficiente. Queda por investigar si (en un ejercicio normativo) el crecimiento pudo haberse acelerado si hubiera sido el caso de que la inversión se concentrara en ramas las más modernas, con mayor crecimiento de la productividad. Probablemente también, en ese caso, la competitividad de la manufactura había sido el soporte para reiniciar las exportaciones sectoriales y acelerar el crecimiento. En todo caso, las condiciones en las que se encontraba la economía exigían que los sectores extractivos proporcionaran los recursos para financiar la inversión industrial.

No obstante, el rápido crecimiento económico y de la industria, no fue suficiente para absorber a la población excedente, de tal manera que las relaciones entre la población total y la población ocupada no se modificó. Por supuesto, este fenómeno es concomitante con la mejoría en las condiciones de vida de la población (por ejemplo, sin modificar la tasa de natalidad, cuando se incrementaron los ingresos, mejoró el sistema de salud y declinó la probabilidad de muerte temprana de los infantes, de modo que la población se expandió) en un país eminentemente rural que se urbanizaba, mientras los hábitos y los valores de la población no necesariamente cambiaron, multiplicándose la población original varias veces antes de estabilizarse, incluso cuando el crecimiento económico tornó en magro. 


\section{Referencias}

Antonelli, G. and N. De Liso (2000) Introduction: An Appraisal of the Economic Analysis of Technological Change. The Path to the Last Decade. En: G. Antonelli and N. De Liso (eds.) Economics of Structural and Technological Change. Londres, Routledge, pp. 12-58.

Aroche Reyes , F. (2005). Desintegración de la estructura productiva mexicana y el empleo. Los coeficientes importantes y su importancia en la integración. Revista Asturiana de Economía(33), 31-66.

Aroche Reyes, F. (2020). On growth regimes, structural change and input coefficients. Economic Systems Research, 1-18.

Avendaño Vargas, B. L., \& Perrotini Hernández, I. (2017). Acumulación de capital, insuficiencia dinámica y crecimiento económico en América Latina. Papeles de Europa, 30(2), 105-124.

Böhm, B., \& Punzo, L. F. (1999). Productivity-Investment Fluctuations and Structural Changes. In L. F. Punzo, Cycles, Growth and Structural Change (pp. 47-92). London: Routledge.

Carter, A. (1970). Structural Change in the American Economy. Harvard University Press.

Fei, J. C., \& Ranis, G. (1961). A Theory of Economic Development. The American Economic Review, 533-565.

Kaldor, N. (1967). Strategic Factors in Economic Development. New York: Cornell University.

Leontief, W. (1963). The Structure of Development. Scientific American, 148-166.

Lewis, W. (1954, Mayo). Economic Development with Unlimited Supplies of Labour. The Manchester School, 22(2).

Lewis, W. A. (1979). The Dual economy revisited. The Manchester School, 211-229.

Smith, A. (1776). An inquiry into the nature and causes of the wealth of nations. Indianapolis: Liberty Classics. 